\title{
Some New Separation Axioms with Application in Dimension Theory
}

\section{Sema Kadhim Jebur}

University of Al-Qadiysia, College of Agriculture Department of Soil and Water Resource

Semh.Alisawi@qu.ed.iq

\author{
Received:-18/1/2017 Accepted:-14/3/2017
}

\begin{abstract}
.
The aim of this paper is to introduce and study the notions of $\theta^{*}$-regular and $\theta^{*}$-normal topological spaces and give some applications in dimension theory.
\end{abstract}

Key Words. $\theta^{*}$-regular, $\theta^{*}$-normal, locally Lindelof, small inductive dimension, large inductive dimension

Physical Classification QA440-699 


\section{0- Introduction.}

The separation axioms is one of the most important in the study of topological spaces and dimension theory. In this paper the notions of $\theta^{*}-$ regular space and $\theta^{*}$-normal are introduces and some properties of such spaces are proved. The $\theta^{*}$ - regular space can be considered as a generalization of $\theta-$ regular space [ $1,2,3]$. The $\theta^{*}$-normal space can be considers as generalization of normal space. Also the notion of $\mathcal{G}-\theta^{*}-$ regular topological space and study some essential properties of this space. This space being the generalization of both $\theta$-regular [1,2,3], and $\mathcal{G}$-regular $[4,5]$.

Moreover we defined the small inductive dimension and large inductive dimension in terms of $\theta^{*}-$ regular and $\theta^{*}$-normal spaces and some essential properties related with small and large inductive dimensions are proved .The concept of dimension is based on the work of Urysohn and Menger. The dimension functions ind, Ind and c.dim were investigated by A.R. Pears in 1975[6]. A topological space is said to be compact $[7,8]$ if collection of that cover.

Theorem 1.3 A topological space $X$ is $\theta^{*}-$ regular if and only if for every basic open covering of $X$ and each $x \in X$ there is an open neighborhood of $x$ its closure can be covered by a countably sub collection of that cover.

Proof. The "only if" part follows from the fact that every basic open cove is an open cover .

The "if" part. Suppose that $\mathcal{U}:=\left\{U_{\lambda}: \lambda \in \Lambda\right\} \quad$ is an open cover of $X$ and $x \in X$. If $\mathcal{B}:=$ $\left\{B_{\gamma}: \gamma \in \Gamma\right\}$ be any open base for $X$,then each $U_{\lambda}$ every open cover admits a finite open subcover . A topological space is said to be locally compact[ $7,8]$ if every point in the space admits a neighborhood with compact closure . A topological space is said to be Lindelof[7,8] if every open cover admits a countable open subcover .

\section{1. $\theta^{*}$ - regular Topological Space}

In this section we introduce the notion of $\theta^{*}-$ regular topological space and study some essential properties of such space .

Definition 1.1. [1,2,3]. A topological space $X$ is said to be $\theta$ - regular if for every open covering of $X$ and each $x \in X$ there is an open neighborhood of $x$ its closure can be covered by a finite sub collection of that cover.

In the following the new definition is introduce.

Definition 1.2. A topological space $X$ is said to be $\theta^{*}-$ regular if for every open covering of $X$ and each $x \in X$ there is an open neighborhood of $x$ its closure can be covered by a countably sub

In the following theorem we characterize the notion of $\theta^{*}-$ regular topological space in terms of basic open cover .

is a union of some members of $\mathcal{B}$ and the totality of all such members of $\mathcal{B}$ is evidently a basic open cover of $X$. By hypothesis there is an open neighborhood $N$ of $x$ such that

$$
\bar{N} \subseteq \cup\left\{B_{\gamma_{n}}: n \in \mathbb{N}\right\}
$$

For each $B_{\gamma_{n}}$ we can select a $U_{\lambda_{n}}$ from $\mathcal{U}$ such that $B_{\gamma_{n}} \subseteq U_{\lambda_{n}}$. It follows that

$$
\bar{N} \subseteq \bigcup\left\{U_{\lambda_{n}}: n \in \mathbb{N}\right\}
$$


Hence $X$ is $\theta^{*}-$ regular

Theorem 1.4 The closed subspace of a $\theta^{*}-$ regular space is $\theta^{*}-$ regular .

Proof. Let $(X, \tau)$ be a $\theta^{*}-$ regular and let $\left(Y, \tau_{Y}\right)$ be a closed subspace of $(X, \tau)$. To show that $\left(Y, \tau_{Y}\right) \quad$ is $\theta^{*}-$ regular. Let $\left\{U_{\lambda}: \lambda \in \Lambda\right\}$ be a $\tau_{Y}$-open cover of $Y$ and $y \in Y$. Then there is a $\tau$-open set $V_{\lambda}$ such that $U_{\lambda}=V_{\lambda} \cap Y$. Hence $\left\{V_{\lambda}: \lambda \in \Lambda\right\} \cup\{X / Y\}$ is a $\tau-$ open cover and $y \in X$. By hypothesis there is a $\tau$-open neighborhood $N$ of $y$ such that

$\left.\bar{N}=\bigcup\left\{U_{\lambda_{n}} \cap \bar{N}: n \in \mathbb{N}\right\}\right) \subseteq \bigcup\left\{U_{\lambda_{n}}: n \in \mathbb{N}\right\}$

This show that $(X, \tau)$ is $\theta^{*}-$ regular

Theorem 1.5 The property of a space being a $\theta^{*}-$ regular is topological property .

Proof. Let $\quad f:(X, \tau) \rightarrow(Y, \mu) \quad$ be homeomorphism function. Suppose that $(X, \tau)$ is $\theta^{*}$ - regular. To show that $(Y, \mu)$ is $\theta^{*}-$ normal. Let $\left\{U_{\lambda}: \lambda \in \Lambda\right\}$ be a $\mu$-open cover of $Y$ and $y \in Y$. Then $\left\{f^{-1}\left(U_{\lambda}\right): \lambda \in \Lambda\right\}$ is a $\tau$-open cover of $X$ and $f^{-1}(y) \in X$. By hypothesis there is a $\tau$-open neighborhood $N$ of $f^{-1}(y)$ such that

$$
\bar{N} \subseteq \bigcup\left\{f^{-1}\left(U_{\lambda_{n}}\right): n \in \mathbb{N}\right\}
$$

Since $f$ is homeomorphism, then $M=f(N)$ is a $\mu$-open neighborhood of $y$ and $f(\bar{N})=\overline{f(N)}=$ $\bar{M}$. Therefore

$$
\bar{M}=f(\bar{N}) \subseteq \bigcup\left\{U_{\lambda_{n}}: n \in \mathbb{N}\right\}
$$

Thus $(Y, \mu)$ is $\theta^{*}-$ regular
Theorem 1.6 The product $X:=\prod_{\lambda \in \Lambda} X_{\lambda}$ is $\theta^{*}-$ regular if and only if each coordinate space $X_{\lambda}$ is $\theta^{*}-$ regular .

Proof. Let each $X_{\lambda}$ is $\theta^{*}-$ regular. Let $x=\left\{x_{\lambda}: \lambda \in \Lambda\right\}$ be any point of the product space $X$ and $\left\{U_{\alpha}: \alpha \in \Delta\right\}$ be any open cover of $X$. Then there exists a basic open set $V_{\alpha}$ in $X$ such that $V_{\alpha} \subseteq U_{\alpha}$. Then $\left\{V_{\alpha}: \alpha \in \Delta\right\}$ is a basic open cover of $X$. Now, $V_{\alpha}=\prod_{\lambda \in \Lambda} V_{(\alpha, \lambda)}$, where $V_{(\alpha, \lambda)}$ is open in $X_{\lambda}$ [ Note that $V_{(\alpha, \lambda)}=X_{\lambda}$ for all but a finite number of $\lambda$ 's but it is immaterial here]. Since $X_{\lambda}$ is $\theta^{*}-$ regular and $\left\{V_{(\alpha, \lambda)}: \alpha \in \Delta\right\}$ is an open cover of $X_{\lambda}$, there exists an open neighborhood $U_{\lambda}$ of $x_{\lambda}$ such that

$$
\bar{U}_{\lambda} \subseteq \bigcup\left\{V_{\left(\alpha_{n}, \lambda\right)}: n \in \mathbb{N}\right\}
$$

Let $=\prod_{\lambda \in \Lambda} U_{\lambda}$, then $\mathcal{W}$ is an open neighborhood of $x$ in $X \quad$.Also $\overline{\mathcal{W}}=\overline{\prod_{\lambda \in \Lambda} U_{\lambda}}=$ $\prod_{\lambda \in \Lambda} \bar{U}_{\lambda}$. Further, since $\bar{U}_{\lambda} \subseteq \bar{V}_{\left(\alpha_{n}, \lambda\right)}$, for every $\lambda \in \Lambda$ and for some $n \in \mathbb{N}$ we have $\overline{\mathcal{W}}=$ $\prod_{\lambda \in \Lambda} \bar{U}_{\lambda} \subseteq \prod_{\lambda \in \Lambda} V_{\left(\alpha_{n}, \lambda\right)}$, for some $n \in \mathbb{N}$. Hence $\bar{W} \subseteq V_{\alpha_{n}}, \quad$ for $\quad$ some $n \in \mathbb{N}$, i.e., $\overline{\mathcal{W}} \subseteq$ $\bigcup\left\{V_{\alpha_{n}}: n \in \mathbb{N}\right\}$. Thus $X$ is $\theta^{*}-$ regular.

Conversely, let the product $X:=\prod_{\lambda \in \Lambda} X_{\lambda} \neq \varnothing$ is $\theta^{*}-$ regular . Let $\gamma \in \Lambda$, we shall show that $X_{\gamma}$ is $\theta^{*}-$ regular . Let $a_{\gamma} \in X_{\gamma}$ and $\left\{G_{(\alpha, \gamma)}: \alpha \in\right.$ $\Delta\}$ be an open cover of $X_{\gamma}$. Since $X \neq \varnothing$, there exists $x=\left\{x_{\lambda}: \lambda \in \Lambda\right\} \in X \quad$ such that $x_{\gamma}=$ $a_{\gamma}$. Let $G_{\alpha}=\pi_{\gamma}^{-1}\left(G_{(\alpha, \gamma)}\right)$, where $\pi_{\gamma}: X \rightarrow X_{\gamma}$ be the projection map. Since $\pi_{\gamma}$ is continuous and onto, then $\left\{G_{\alpha}: \alpha \in \Delta\right\}$ is an open cover of $X$. Since $X$ is $\theta^{*}-$ regular, there exists a basic neighborhood $N:=\prod_{\lambda \in \Lambda} N_{\lambda}$ of $x$ such that $\bar{N} \subseteq \bigcup\left\{G_{\alpha_{n}}: n \in \mathbb{N}\right\}$. Then $x_{\gamma} \in N_{\gamma}$. Since 
$\bar{N}=\overline{\prod_{\lambda \in \Lambda} N_{\lambda}}=\prod_{\lambda \in \Lambda} \bar{N}_{\lambda}$, then $\bar{N}=\prod_{\lambda \in \Lambda} \bar{N}_{\lambda} \subseteq$ $\bigcup\left\{G_{\alpha_{n}}: n \in \mathbb{N}\right\}$. Then $\bar{N}_{\gamma} \subseteq \cup\left\{G_{\left(\alpha_{n}, \gamma\right)}: n \in \mathbb{N}\right\}$. Thus for every open cover $\left\{G_{(\alpha, \gamma)}: \alpha \in \Delta\right\}$ of $X_{\gamma}$ and each $a_{\gamma} \in X_{\gamma}$, there exists an open neighborhood $N_{\gamma}$ of $a_{\gamma}$ such that $\bar{N}_{\gamma} \subseteq$ $\mathrm{U}\left\{G_{\left(\alpha_{n}, \gamma\right)}: n \in \mathbb{N}\right\}$. Hence $X_{\gamma}$ is $\theta^{*}-$ regular

Definition $1.7[7,8]$ A topological space $X$ is said to be locally compact (Lindelof) space if every point of $X$ admits an open neighborhood with compact (lindelof) closure .

Theorem 1.8 Every locally Lindelöff space is $\theta^{*}-$ regular .

Proof. Suppose that $(X, \tau)$ is locally Lindelöff space. Let $\left\{U_{\lambda}: \lambda \in \Lambda\right\}$ be a $\tau$-open cover of $X$ and

Example 1.12 The real line is $\theta^{*}$-regular since the real line is locally compact .

\section{2. $\mathcal{G}-\theta^{*}$-regular topological space}

In this section we introduce the notion of $\mathcal{G}-\theta^{*}$-regular topological space and study some essential properties of this space. This space being the generalization of both $\theta$-regular [1,2,3], and $\mathcal{G}$-regular [4,5] .

Definition 2.1 Let $(X, \tau)$ be a topological space and $\mathcal{G}$ be a grill on $X$. Then $X$ is said to be $\mathcal{G}-\theta^{*}$-regular if for every open covering $\mathcal{U}=\left\{U_{\lambda}: \lambda \in \Lambda\right\}$ of $X$ and each $x \in X$ there is an open neighborhood $V$ of $x$ and a countable subcollection $\mathcal{U}=\left\{U_{\lambda_{n}}: n \in \mathbb{N}\right\}$ such that

$$
\bar{V} / \bigcup\left\{U_{\lambda_{n}}: n \in \mathbb{N}\right\} \notin \mathcal{G} .
$$

$x \in X$. By hypothesis there exists a $\tau$-open neighborhood $N$ of $x$ such that $\bar{N}$ is Lindelöff. Since $\left\{U_{\lambda} \cap \bar{N}: \lambda \in \Lambda\right\}$ is $\tau_{\bar{N}}$-open cover of $\bar{N}$, by Lindelöffness of $\bar{N}$ we have

$$
\left.\bar{N}=\bigcup\left\{U_{\lambda_{n}} \cap \bar{N}: n \in \mathbb{N}\right\}\right) \subseteq \bigcup\left\{U_{\lambda_{n}}: n \in \mathbb{N}\right\}
$$

This show that $(X, \tau)$ is $\theta^{*}$ - regular

Corollary 1.9 Every locally compact space is $\theta^{*}-$ regular.

Corollary 1.10 Every compact (Lindelof) space is $\theta^{*}-$ regular .

Corollary 1.11 Every second countable space is $\theta^{*}$-regular .

Proposition 2.2 Every $\theta^{*}$ - regular space $(X, \tau)$ is $\mathcal{G}-\theta^{*}$-regular for every grill $\mathcal{G}$ on $X$.

Proof. Suppose that $(X, \tau)$ is $\theta^{*}-$ regular space. Let $\mathcal{G}$ be any grill on $X, U=\left\{U_{\lambda}: \lambda \in \Lambda\right\}$ be any open cover of $X$ and $x \in X$. By hypothesis there is an open neighborhood $V$ of $x$ and a countable subcollection $U=\left\{U_{\lambda_{n}}: n \in \mathbb{N}\right\}$ such that

$$
\bar{V} \subseteq \cup\left\{U_{\lambda_{n}}: n \in \mathbb{N}\right\}
$$

Then

$$
\bar{V} / \bigcup\left\{U_{\lambda_{n}}: n \in \mathbb{N}\right\}=\emptyset
$$

But $\mathcal{G}$ is a non-null collection of nonempty set, so $\bar{V} \subseteq \cup\left\{U_{\lambda_{n}}: n \in \mathbb{N}\right\} \notin \mathcal{G}$. Hence $(X, \tau)$ is $\mathcal{G}-\theta^{*}$-regular for every grill $\mathcal{G}$ on $X$. 
Theorem 2.3 Let $f:(X, \tau) \rightarrow(Y, \mu)$ be homeomorphism function. Let $\mathcal{G}$ be a grill on $X$. If $:(X, \tau)$ is $\mathcal{G}-\theta^{*}$-regular, then $(Y, \mu)$ is $\mathcal{G}^{\prime}-\theta^{*}$-regular, where $\mathcal{G}^{\prime}=f[\mathcal{G}]$.

Proof. Let $f:(X, \tau) \rightarrow(Y, \mu)$ be homeomorphism function. First, since $f$ is bijective, then $\mathcal{G}^{\prime}=f[\mathcal{G}]$ is grill on $Y$.Suppose that $(X, \tau)$ is $\theta^{*}-$ regular. To show that $(Y, \mu)$ is $\theta^{*}-$ regular. Let $\left\{U_{\lambda}: \lambda \in \Lambda\right\}$ be a $\mu$-open cover of $Y$ and $y \in Y$. Then $\left\{f^{-1}\left(U_{\lambda}\right): \lambda \in \Lambda\right\}$ is a $\tau$-open cover of $X$ and $f^{-1}(y) \in X$. By hypothesis there is a $\tau$-open neighborhood $N$ of $f^{-1}(y)$ such that

$$
\bar{N} / \bigcup\left\{f^{-1}\left(U_{\lambda_{n}}\right): n \in \mathbb{N}\right\} \notin \mathcal{G} .
$$

Since $f$ is homeomorphism, then $M=f(N)$ is a $\mu$-open neighborhood of $y$ and $f(\bar{N})=\overline{f(N)}=$ $\bar{M}$. Now

$$
\begin{aligned}
& f\left[\bar{N} / \bigcup\left\{f^{-1}\left(U_{\lambda_{n}}\right): n \in \mathbb{N}\right\}\right]= \\
& f[\bar{N}] / f\left[\cup\left\{f^{-1}\left(U_{\lambda_{n}}\right): n \in \mathbb{N}\right\}\right] \notin f[\mathcal{G}] .
\end{aligned}
$$

That is,

$$
\bar{M} /\left\{U_{\lambda_{n}}: n \in \mathbb{N}\right\} \notin \mathcal{G}^{\prime}
$$

Thus $(Y, \mu)$ is $\mathcal{G}^{\prime}-\theta^{*}-$ regular.

Theorem 2.4 Let $\mathcal{G}=\prod_{\lambda \in \Lambda} \mathcal{G}_{\lambda}$ where $\mathcal{G}_{\lambda}$ is grill on $X_{\lambda}$. Then the product $X:=\prod_{\lambda \in \Lambda} X_{\lambda}$ is $\mathcal{G}-\theta^{*}-$ regular if and only if each coordinate space $X_{\lambda}$ is $\mathcal{G}_{\lambda}-\theta^{*}-$ regular .

Proof. Let each $X_{\lambda}$ is $\theta^{*}-$ regular . Let $x=$ $\left\{x_{\lambda}: \lambda \in \Lambda\right\}$ be any point of the product space $X$ and $\left\{U_{\alpha}: \alpha \in \Delta\right\}$ be any open cover of $X$. Then there exists a basic open set $V_{\alpha}$ in $X$ such that $V_{\alpha} \subseteq U_{\alpha}$.
Then $\left\{V_{\alpha}: \alpha \in \Delta\right\}$ is a basic open cover of $X$. Now, $V_{\alpha}=\prod_{\lambda \in \Lambda} V_{(\alpha, \lambda)}$, where $V_{(\alpha, \lambda)}$ is open in $X_{\lambda}$ [ Note that $V_{(\alpha, \lambda)}=X_{\lambda}$ for all but a finite number of $\lambda$ 's but it is immaterial here] . Since $X_{\lambda}$ is $\mathcal{G}_{\lambda}-\theta^{*}-$ regular and $\left\{V_{(\alpha, \lambda)}: \alpha \in \Delta\right\}$ is an open cover of $X_{\lambda}$ ,there exists an open neighborhood $U_{\lambda}$ of $x_{\lambda}$ such that

$$
\bar{U}_{\lambda} / \cup\left\{V_{\left(\alpha_{n}, \lambda\right)}: n \in \mathbb{N}\right\} \notin \mathcal{G}_{\lambda}
$$

Let $=\prod_{\lambda \in \Lambda} U_{\lambda}$, then $\mathcal{W}$ is an open neighborhood of $x$ in $X$. Also $\overline{\mathcal{W}}=\overline{\prod_{\lambda \in \Lambda} U_{\lambda}}=\prod_{\lambda \in \Lambda} \bar{U}_{\lambda}$. Further, since $\bar{U}_{\lambda} \subseteq \bar{V}_{\left(\alpha_{n}, \lambda\right)}$, for every $\lambda \in \Lambda$ and for some $n \in \mathbb{N}$ we have $\bar{W}=\prod_{\lambda \in \Lambda} \bar{U}_{\lambda} \subseteq \prod_{\lambda \in \Lambda} V_{\left(\alpha_{n}, \lambda\right)}$, for some $n \in \mathbb{N}$. Hence $\overline{\mathcal{W}} \subseteq V_{\alpha_{n}}$, for some $n \in \mathbb{N}$, i.e., $\overline{\mathcal{W}} / \cup\left\{V_{\alpha_{n}}: n \in \mathbb{N}\right\} \notin \mathcal{G}$. Thus $X$ is $\theta^{*}-$ regular.

Conversely, let the product $X:=\prod_{\lambda \in \Lambda} X_{\lambda} \neq \emptyset$ is $\theta^{*}-$ regular . Let $\gamma \in \Lambda$, we shall show that $X_{\gamma}$ is $\theta^{*}-$ regular . Let $a_{\gamma} \in X_{\gamma}$ and $\left\{G_{(\alpha, \gamma)}: \alpha \in \Delta\right\}$ be an open cover of $X_{\gamma}$. Since $X \neq \emptyset$, there exists $x=\left\{x_{\lambda}: \lambda \in \Lambda\right\} \in X$ such that $x_{\gamma}=a_{\gamma} . \quad$ Let $G_{\alpha}=\pi_{\gamma}^{-1}\left(G_{(\alpha, \gamma)}\right)$, where $\pi_{\gamma}: X \rightarrow X_{\gamma}$ be the projection map. Since $\pi_{\gamma}$ is continuous and onto, then $\left\{G_{\alpha}: \alpha \in \Delta\right\}$ is an open cover of . Since $X$ is $\theta^{*}-$ regular, there exists a basic neighborhood $N:=\prod_{\lambda \in \Lambda} N_{\lambda} \quad$ of $x$ such that $\bar{N} \subseteq \bigcup\left\{G_{\alpha_{n}}: n \in\right.$ $\mathbb{N}\}$. Then $x_{\gamma} \in N_{\gamma}$. Since $\bar{N}=\overline{\prod_{\lambda \in \Lambda} N_{\lambda}}=$ $\prod_{\lambda \in \Lambda} \bar{N}_{\lambda}$, then $\bar{N}=\prod_{\lambda \in \Lambda} \bar{N}_{\lambda} \subseteq \bigcup\left\{G_{\alpha_{n}}: n \in \mathbb{N}\right\}$. Then $\bar{N}_{\gamma} \subseteq \cup\left\{G_{\left(\alpha_{n}, \gamma\right)}: n \in \mathbb{N}\right\}$. Thus for every open cover $\left\{G_{(\alpha, \gamma)}: \alpha \in \Delta\right\}$ of $X_{\gamma}$ and each $a_{\gamma} \in$ $X_{\gamma}$, there exists an open neighborhood $N_{\gamma}$ of $a_{\gamma}$ such that $\bar{N}_{\gamma} \subseteq \cup\left\{G_{\left(\alpha_{n}, \gamma\right)}: n \in \mathbb{N}\right\}$. Hence $X_{\gamma}$ is $\theta^{*}-$ regular 


\section{3. $\theta^{*}$ - normal Topological Space}

In this section we introduce the notion of $\theta^{*}-$ normal topological space and study some essential properties of such space .

Definition 3.1. A topological space $X$ is said to be $\theta^{*}$-normal if for every open covering of $X$ and each closed set $F$ in $X$ there is an open neighborhood of $F$ its closure can be covered by a countably sub collection of that cover.

and by hypothesis there exists an open neighborhood $N$ of $\{x\}$ such that $\bar{N} \subseteq$ $\bigcup\left\{U_{\lambda_{n}}: n \in \mathbb{N}\right\}$. Thus $X$ is $\theta^{*}$-regular

Theorem 3.3 A topological space $X$ is $\theta^{*}-$ normal if and only if for every basic open covering of $X$ and each closed set $F$ in $X$ there is an open neighborhood of $x$ its closure can be covered by a countably infinite sub collection of that cover.

$$
\bar{N} \subseteq \bigcup\left\{B_{\gamma_{n}}: n \in \mathbb{N}\right\}
$$

For each $B_{\gamma_{n}}$ we can select a $U_{\lambda_{n}}$ from $\mathcal{U}$ such that $B_{\gamma_{n}} \subseteq U_{\lambda_{n}}$. It follows that

$$
\bar{N} \subseteq \bigcup\left\{U_{\lambda_{n}}: n \in \mathbb{N}\right\}
$$

Hence $X$ is $\theta^{*}-$ normal

Theorem 3.4 The closed subspace of a $\theta^{*}-$ normal space is $\theta^{*}-$ normal.

Proof Let $(X, \tau)$ be a $\theta^{*}-$ normal and let $\left(Y, \tau_{Y}\right)$ be a subspace of $(X, \tau)$. To show that $\left(Y, \tau_{Y}\right)$ is $\theta^{*}-$ normal . Let $\left\{U_{\lambda}: \lambda \in \Lambda\right\}$ be a $\tau_{Y}$-open $\bar{M}=\bar{N} \cap Y \subseteq\left[\bigcup\left\{V_{\lambda_{n}}: n \in \mathbb{N}\right\} \cup\{X / Y\}\right] \cap Y$

$$
=\bigcup\left\{V_{\lambda_{n}} \cap Y: n \in \mathbb{N}\right\}=\bigcup\left\{U_{\lambda_{n}}: n \in \mathbb{N}\right\}
$$

Proposition 3.2 If a $T_{1}$-space is $\theta^{*}$-normal, then it is $\theta^{*}$-regular.

Proof Suppose that $X$ be a $\theta^{*}$-normal topological space . Let $\mathcal{U}:=\left\{U_{\lambda}: \lambda \in \Lambda\right\}$ be an open cover of $X$ and $x \in X$. Since $X$ is $T_{1}$-space, then every singleton set is closed .

Thus $\{x\}$ is closed set

Proof The "only if" part follows from the fact that every basic open cove is an open cover .

The "if" part . Suppose that $\mathcal{U}:=\left\{U_{\lambda}: \lambda \in \Lambda\right\}$ is an open cover of $X$ closed set $F$ in $X$. If $\mathcal{B}:=$ $\left\{B_{\gamma}: \gamma \in \Gamma\right\}$ be any open base for $X$, then each $U_{\lambda}$ is a union of some members of $\mathcal{B}$ and the totality of all such members of $\mathcal{B}$ is evidently a basic open cover of $X$. By hypothesis there is an open neighborhood $N$ of $F$ such that

cover of $Y$ and $F$ be a $\tau_{Y}-$ closed. Then there is a $\tau$-open set $V_{\lambda}$ such that $U_{\lambda}=V_{\lambda} \cap Y$. Hence $\left\{V_{\lambda}: \lambda \in \Lambda\right\} \cup\{X / Y\}$ is a $\tau$ - open cover and there exists a $\tau$-closed set $K$. By hypothesis there is a $\tau$-open neighborhood $N$ of $K$ such that a

$$
\bar{N} \subseteq \bigcup\left\{V_{\lambda_{n}}: n \in \mathbb{N}\right\} \cup\{X / Y\} .
$$

Then $M=N \cap Y$ is a $\tau_{Y}$-open neighborhood of $F$ and $\bar{M}=\bar{N} \cap Y \quad$. Now, 
Thus $\left(Y, \tau_{Y}\right)$ is $\theta^{*}-$ normal.

Theorem 3.5 The property of a space being a $\theta^{*}-$ normal is topological property .

Proof Let

$$
f:(X, \tau) \rightarrow(Y, \mu) \quad \text { be }
$$

homeomorphism function. Suppose that $(X, \tau)$ is

$$
\bar{N} \subseteq \bigcup\left\{f^{-1}\left(U_{\lambda_{n}}\right): n \in \mathbb{N}\right\}
$$

Since $f$ is homeomorphism, then $M=f(N)$ is a $\mu$-open neighborhood of $y$ and $f(\bar{N})=\overline{f(N)}=$ $\bar{M}$. Therefore

$$
\bar{M}=f(\bar{N}) \subseteq \bigcup\left\{U_{\lambda_{n}}: n \in \mathbb{N}\right\} .
$$

Thus $(Y, \mu)$ is $\theta^{*}-$ normal

Theorem 3.6 Every locally Lindelöff space is $\theta^{*}-$ normal .

Proof Suppose that $(X, \tau)$ is locally Lindelöff space . Let $\left\{U_{\lambda}: \lambda \in \Lambda\right\}$ be a $\tau$-open cover of $X$ and $F$ be a $\tau$-closed set . By hypothesis there exists a $\tau$-open neighborhood $N$ of $F$ such that $\bar{N}$ is Lindelöff. Since $\left\{U_{\lambda} \cap \bar{N}: \lambda \in \Lambda\right\}$ is $\tau_{\bar{N}}$-open cover of $\bar{N}$, by Lindelöffness of $\bar{N}$ we have

$$
\left.\bar{N}=\bigcup\left\{U_{\lambda_{n}} \cap \bar{N}: n \in \mathbb{N}\right\}\right) \subseteq \bigcup\left\{U_{\lambda_{n}}: n \in \mathbb{N}\right\}
$$

This show that $(X, \tau)$ is $\theta^{*}-$ normal

$X_{\lambda}$ is $\theta^{*}-$ regular and $\left\{V_{(\alpha, \lambda)}: \alpha \in \Delta\right\}$ is an open cover of $X_{\lambda}$, there exists an open neighborhood $U_{\lambda}$ of $F_{\lambda}$ such that

$$
\bar{U}_{\lambda} \subseteq \bigcup\left\{V_{\left(\alpha_{n}, \lambda\right)}: n \in \mathbb{N}\right\}
$$

$\theta^{*}-$ regular. To show that $(Y, \mu)$ is $\theta^{*}-$ normal . Let $\left\{U_{\lambda}: \lambda \in \Lambda\right\}$ be a $\mu$-open cover of $Y$ and $F$ be a $\mu-$ closed set. Then $\left\{f^{-1}\left(U_{\lambda}\right): \lambda \in \Lambda\right\}$ is a $\tau$-open cover of $X$ and $f^{-1}(F)$ is $\tau$-closed set . By hypothesis there is a $\tau$-open neighborhood $N$ of $f^{-1}(F)$ such that

Theorem 3.7 The product $X:=\prod_{\lambda \in \Lambda} X_{\lambda}$ is $\theta^{*}-$ normal if and only if each coordinate space $X_{\lambda}$ is $\theta^{*}-$ normal .

Proof Let each $X_{\lambda}$ is $\theta^{*}-$ normal. Let $F=\prod\left\{F_{\lambda}: \lambda \in \Lambda\right\}$ be any closed subset of the product space $X$ and $\left\{U_{\alpha}: \alpha \in \Delta\right\}$ be any open cover of $X$. Then there exists a basic open set $V_{\alpha}$ in $X$ such that $V_{\alpha} \subseteq U_{\alpha}$. Then $\left\{V_{\alpha}: \alpha \in \Delta\right\}$ is a basic open cover of $X$. Now, $V_{\alpha}=$ $\prod_{\lambda \in \Lambda} V_{(\alpha, \lambda)}$, where $V_{(\alpha, \lambda)}$ is open in $X_{\lambda}$ [ Note that $V_{(\alpha, \lambda)}=X_{\lambda}$ for all but a finite number of $\lambda$ 's but it is immaterial here] Since

Let $W=\prod_{\lambda \in \Lambda} U_{\lambda}$ then $W$ is an open neighborhood of $F$ in $X$. Also $\bar{W}=\overline{\prod_{\lambda \in \Lambda} U_{\lambda}}=$ $\prod_{\lambda \in \Lambda} \bar{U}_{\lambda}$. Further, since $\bar{U}_{\lambda} \subseteq \bar{V}_{\left(\alpha_{n}, \lambda\right)}$, for every $\lambda \in \Lambda$ and for some $n \in \mathbb{N}$ we have $\bar{W}=\prod_{\lambda \in \Lambda} \bar{U}_{\lambda} \subseteq \prod_{\lambda \in \Lambda} V_{\left(\alpha_{n}, \lambda\right)}$, for some $n \in \mathbb{N}$. 
Hence $\bar{W} \subseteq V_{\alpha_{n}}$, for some $n \in \mathbb{N}$, i.e., $\bar{W} \subseteq$ $\bigcup\left\{V_{\alpha_{n}}: n \in \mathbb{N}\right\}$. Thus $X$ is $\theta^{*}-$ normal .

Conversely, let the product $X:=\prod_{\lambda \in \Lambda} X_{\lambda} \neq \emptyset$ is $\theta^{*}-$ regular. Let $\gamma \in \Lambda$. We shall show that $X_{\gamma}$ is $\theta^{*}$-normal. Let $a_{\gamma} \in X_{\gamma}$ and $\left\{G_{(\alpha, \gamma)}: \alpha \in \Delta\right\}$ be an open cover of $X_{\gamma}$. Since $X \neq \emptyset$, there exists a closed subset $F=\left\{F_{\lambda}: \lambda \in \Lambda\right\}$ of $X$ such that $F_{\gamma}=A_{\gamma}$. Let $G_{\alpha}=\pi_{\gamma}^{-1}\left(G_{(\alpha, \gamma)}\right)$, where $\pi_{\gamma}: X \rightarrow X_{\gamma}$ be the projection map . Since $\pi_{\gamma}$ is continuous and onto, then $\left\{G_{\alpha}: \alpha \in \Delta\right\}$ is an open cover of $X$. Since $X$ is $\theta^{*}-$ normal, there exists a basic neighborhood $N:=\prod_{\lambda \in \Lambda} N_{\lambda}$ of $F$ such that $\bar{N} \subseteq \bigcup\left\{G_{\alpha_{n}}: n \in \mathbb{N}\right\}$. Then $F_{\gamma} \subseteq N_{\gamma}$. Since $\bar{N}=\overline{\prod_{\lambda \in \Lambda} N_{\lambda}}=\prod_{\lambda \in \Lambda} \bar{N}_{\lambda}$, then $\bar{N}=\prod_{\lambda \in \Lambda} \bar{N}_{\lambda} \subseteq$ $\bigcup\left\{G_{\alpha_{n}}: n \in \mathbb{N}\right\}$. Then $\quad \bar{N}_{\gamma} \subseteq \bigcup\left\{G_{\left(\alpha_{n}, \gamma\right)}: n \in\right.$ $\mathbb{N}\}$. Thus for every open cover $\left\{G_{(\alpha, \gamma)}: \alpha \in \Delta\right\}$ of $X_{\gamma}$ and each $A_{\gamma} \subseteq X_{\gamma}$, there exists an open neighborhood $N_{\gamma}$ of $A_{\gamma}$ such that $\bar{N}_{\gamma} \subseteq$ $\bigcup\left\{G_{\left(\alpha_{n}, \gamma\right)}: n \in \mathbb{N}\right\}$. Hence $X_{\gamma}$ is $\theta^{*}-$ normal .

\section{4- Application in Dimension Theory}

" In this section we give some applications of $\theta^{*}$-regular and $\theta^{*}$-normal spaces in dimension theory.

Definition 4.1 Let $X$ be a topological space .

The following conditions define the $\theta^{*}-$ small inductive dimension of $X$ :

(i) $\theta^{*}-$ ind $X=-1$ iff $=\varnothing$,

(ii) $\theta^{*}-$ ind $X \leq n$ if for every open covering of $X$ and each $x \in X$ there is an open neighborhood $N$ of $x$ can be covered by countably infinite subcollection of that cover and ) $\theta^{*}-$ ind $\partial(N) \leq$ $n-1$,

(iii) $\theta^{*}-$ ind $X=n$ if $\theta^{*}-$ ind $X \leq n$ and the inequality $\theta^{*}-$ ind $X \leq n-1$ does not hold,

(iv) $\theta^{*}-$ ind $X=\infty$ if $\theta^{*}-$ ind $X=n$ for no $n$.

Theorem 4.2 Let $X$ be a topological space . If $\theta^{*}-$ ind $X=0$, then $X$ is $\theta^{*}$-regular .

Proof Suppose that $\theta^{*}-$ ind $X=0$. Let $x \in X$ and let $\Psi=\left\{U_{\lambda}: \lambda \in \Lambda\right\}$ be an open covering of $X$. By hypothesis there is an open neighborhood $N$ of $x$ such that

$$
N \subset U_{\lambda \in \gamma} U_{\lambda}
$$

where $\gamma$ is countably infinite subset of $\Lambda$, and $\theta^{*}-$ ind $\partial(N) \leq-1$. Hence $\quad \partial(N)=\emptyset$.

Therefore $N$ is open and closed set and consequently $N=\bar{N}$. Then $\bar{N} \subset \bigcup_{\lambda \in \gamma} U_{\lambda}$ where $\gamma$ is countably infinite subset of $\Lambda$. Therefore $X$ is $\theta^{*}$-regular

Theorem 4.3 If $\left(Y, \tau_{Y}\right)$ be a closed subspace of a topological space $(X, \tau)$, then

$$
\theta^{*}-\text { ind } Y \leq \theta^{*}-\text { ind } X
$$

Proof It is enough to show that if $\theta^{*}-$ ind $X \leq$ $n$ then $\theta^{*}-$ ind $Y \leq n$. Suppose that $\theta^{*}-$ ind $X \leq n$. We shall prove by induction on $\theta^{*}-$ ind $X=n$.If $\theta^{*}-$ ind $X=-1$, then it is obviously true. Suppose that the statement is true for $n-1$, to prove it is true for $n$. Let $y \in Y$ and let $\Psi=\left\{U_{\lambda}: \lambda \in \Lambda\right\}$ be a $\tau_{Y}$-open cover of 
$Y$. Then there exists a $\tau-$ open set $V_{\lambda}$ such that $U_{\lambda}=V_{\lambda} \cap Y$. The collection

$$
\Pi=\left\{V_{\lambda}: \lambda \in \Lambda\right\} \cup\{\mathrm{X} / \mathrm{Y}\}
$$

form a $\tau$-open cover of $X$. Since $y \in X$ and $\theta^{*}-$ ind $X \leq n$, then there is a $\tau$-open neighborhood $M$ of $y$ such that

ably infinite subset of $\Lambda$. Since $\partial_{Y}(N) \subset$ $\partial(M) \cap Y$, then $\partial_{Y}(N) \subset \partial(M)$.

But $\theta^{*}-\operatorname{ind} \partial(M) \leq n-1$, therefore $\theta^{*}-$ ind $\partial(N) \leq n-1$. Thus $\theta^{*}-$ ind $Y \leq n-$ 1

Theorem 4.4 If the two spaces $X$ and $Y$ are homeomorphic space, then $\theta^{*}-$ ind $X=\theta^{*}-$ ind $Y$.

Proof Suppose that $X$ and $Y$ are two homeomorphic spaces and $f$ be a homeomorphism from $X$ onto $Y$. We shall prove by induction .

If $\theta^{*}-$ ind $X=-1$, then $X=\emptyset$. Since $f$ is homeomorphism then $Y=\emptyset$. Hence $\theta^{*}-$ ind $Y=-1$. Suppose that $\theta^{*}-$ ind $X=\theta^{*}-$ ind $Y$ for $n-1$. To show it is true for $n$. Suppose that $\theta^{*}-$ ind $X=n$. Let $y \in Y$ and let $\Phi=\left\{U_{\lambda}: \lambda \in \Lambda\right\}$ be an open cover of $Y$. Then $x=f^{-1}(y)$ is a point in $X$ and the collection

$$
\Pi=\left\{f^{-1}\left[U_{\lambda}\right]: \lambda \in \Lambda\right\}
$$

form an open cover of $X$. Since $\theta^{*}-$ ind $X=$ $n$, then there exists an open neighborhood $N$ of $x$ such that $N \subset U_{\lambda \in \gamma} f^{-1}\left[U_{\lambda}\right]$, where $\gamma$ is a countably infinite subset of $\Lambda$ and $\theta^{*}-$

$$
M \subset U_{\lambda \in \gamma} V_{\lambda}
$$

where $\gamma$ is countably infinite subset of $\Lambda$ and $\theta^{*}-\operatorname{ind} \partial(M) \leq n-1$. Thus

$M \cap Y \subset \bigcup_{\lambda \in \gamma}\left(V_{\lambda} \cap Y\right)=\bigcup_{\lambda \in \gamma} U_{\lambda}$.

Hence $N=M \cap Y$ is $\tau_{Y}$-open neighborhood of $y$ and $N \subset \cup_{\lambda \in \gamma} U_{\lambda}$, where $\gamma$ is count $\operatorname{ind} \partial(N)=n-1$. Therefore $f[N]$ be an open neighborhood of $y \in Y$ and

$$
f[N] \subset \bigcup_{\lambda \in \gamma} U_{\lambda}
$$

where $\gamma$ is a countably infinite subset of $\Lambda$ with $\theta^{*}-\operatorname{ind} \partial(f[N])=n-1$. This complete the proof

Definition 4.5 Let $X$ be a topological space . The following conditions define the $\theta^{*}-$ large inductive dimension of $X$ :

(i) $\theta^{*}-$ Ind $X=-1$ iff $X=\emptyset$.

(ii) $\theta^{*}-$ Ind $X \leq n$ if for every open covering of $X$ and each closed set $F$ in $X$ there is an open neighborhood $N$ of $F$ can be covered by countably infinite subcollection of that cover and $\theta^{*}-$ $\operatorname{Ind} \partial(N) \leq n-1$,

(iii) $\theta^{*}-$ Ind $X=n$ if $\theta^{*}-\operatorname{Ind} X \leq n$ and the inequality $\theta^{*}-$ Ind $X \leq n-1$ does not hold,

(iv) $\theta^{*}-$ Ind $X=\infty$ if $\theta^{*}-$ Ind $X=n$ for no $n$.

Theorem 4.6 Let $X$ be a topological space . If $\theta^{*}-$ Ind $X=0$, then $X$ is $\theta^{*}$-normal .

Proof Suppose that $\theta^{*}-\operatorname{Ind} X=0$. Let closed set $F$ in $X$ and let 
$\Psi=\left\{U_{\lambda}: \lambda \in \Lambda\right\}$ be an open covering of $X$. By hypothesis there is an open neighborhood $N$ of $F$ such that

where $\gamma$ is countably infinite subset of $\Lambda$, and $\theta^{*}-\operatorname{Ind} \partial(N) \leq-1$. Hence $\quad \partial(N)=\emptyset$.

Therefore $N$ is open and closed set and

Corollary 4.7 Let $X$ be a $T_{1}-$ space. If $\theta^{*}-$ Ind $X=0$, then $X$ is $\theta^{*}$-regular .

Proof Combine Proposition(3.2) and Theorem (4.6).

Theorem 4.8 If $\left(Y, \tau_{Y}\right)$ be a closed subspace of a topological space $(X, \tau)$, then then $\theta^{*}-$ Ind $X \leq \theta^{*}-$ Ind $Y$.

Proof It is enough to show that if $\theta^{*}-$ Ind $X \leq$ $n$, then $\theta^{*}-$ Ind $Y \leq n$. Suppose that $\theta^{*}-$ Ind $X \leq n$. We shall prove by induction on $\theta^{*}-$ Ind $X=n$. If $\theta^{*}-\operatorname{Ind} X=-1$, then it is obviously true. Suppose that the statement is true for $n-1$, to prove it is true for $n$. Let $F$ be a closed set in $Y$ and let $\Psi=\left\{U_{\lambda}: \lambda \in \Lambda\right\}$ be a $\tau_{Y}$-open cover of $Y$. Then there exists a $\tau$-open set $V_{\lambda}$ such that $U_{\lambda}=V_{\lambda} \cap Y$. The collection

$$
\Pi=\left\{V_{\lambda}: \lambda \in \Lambda\right\} \cup\{\mathrm{X} / \mathrm{Y}\}
$$

form a $\tau$ - open cover of $X$. Since $F$ is closed in $Y$ and $Y$ is closed then $F$ is closed in $X$. By hypothesis $\theta^{*}-$ Ind $X \leq n$, then there is a $\tau$-open neighborhood $M$ of $F$ such that

$$
M \subset U_{\lambda \in \gamma} V_{\lambda}
$$

$$
N \subset \mathrm{Y}_{\lambda \in \gamma} U_{\lambda}
$$

consequently $\quad N=\bar{N}$. Then $\bar{N} \subset \bigcup_{\lambda \in \gamma} U_{\lambda}$ where $\gamma$ is countably infinite subset of $\Lambda$. Therefore $X$ is $\theta^{*}$-normal

where $\gamma$ is countably infinite subset of $\Lambda$ and $\theta^{*}-\operatorname{Ind} \partial(M) \leq n-1$. Thus

$$
M \cap Y \subset \bigcup_{\lambda \in \gamma}\left(V_{\lambda} \cap Y\right)=\bigcup_{\lambda \in \gamma} U_{\lambda} .
$$

Hence $N=M \cap Y$ is $\tau_{Y}$-open neighborhood of $F$ and $N \subset \cup_{\lambda \in \gamma} U_{\lambda}$, where $\gamma$ is countably infinite subset of $\Lambda$. Since $\partial_{Y}(N) \subset \partial(M) \cap Y$ , then $\partial_{Y}(N) \subset \partial(M)$. But $\theta^{*}-\operatorname{Ind} \partial(M) \leq$ $n-1$, therefore $\theta^{*}-\operatorname{Ind} \partial(N) \leq n-1$. Thus $\theta^{*}-$ Ind $Y=n$.

Theorem 4.9 If the two spaces $X$ and $Y$ are homeomorphic space, then $\theta^{*}-$ Ind $X=\theta^{*}-$ Ind $Y$.

Proof Suppose that $X$ and $Y$ are two homeomorphic spaces and $f$ be a homeomorphism from $X$ onto $Y$. We shall prove by induction .

$$
\text { If } \theta^{*}-\text { Ind } X=-1 \text {, then } X=\emptyset \quad \text {. Since } f
$$
is homeomorphism then $Y=\emptyset$. Hence $\theta^{*}-$ Ind $Y=-1$. Suppose that $\theta^{*}-$ Ind $X=$ $\theta^{*}-$ Ind $Y$ for $n-1$. To show it is true for $n$ - Suppose that $\theta^{*}-\operatorname{Ind} X=n$. Let $F$ closed set in $Y$ and let $\Psi=\left\{U_{\lambda}: \lambda \in \Lambda\right\}$ be an open cover of $Y$. Then $E=f^{-1}[F]$ is a closed set in $X$ and the collection

$$
\Pi=\left\{f^{-1}\left[U_{\lambda}\right]: \lambda \in \Lambda\right\}
$$


form an open cover of $X$. Since $\theta^{*}-$ Ind $X=$ $n$,then there exists an open neighborhood $N$ of $E$ such that $N \subset \cup_{\lambda \in \gamma} f^{-1}\left[U_{\lambda}\right]$,

where $\gamma$ is a countably infinite subset of $\Lambda$ and $\theta^{*}-\operatorname{Ind} \partial(N) \leq n-1$. Therefore $f[N]$ be an open neighborhood of $F$ in $Y$ and $f[N] \subset$ $\mathrm{U}_{\lambda \in \gamma} U_{\lambda}$, where $\gamma$ is a countably infinite subset of $\Lambda$ with $\theta^{*}-\operatorname{Ind} \partial(N)=n-1$, which completes the proof

Theorem 4.10 For every $T_{1}-$ space $X$ we have $\theta^{*}-$ ind $X \leq \theta^{*}-$ Ind $X$.

Proof Let us show by induction on the number $n$ that $\theta^{*}-$ Ind $X \leq n$ implies $\theta^{*}-$ ind $X \leq n$ . Since the validity of the assertion for $n=-1$, we shall assume it for $\theta^{*}-$ Ind $X \leq n$. Now, assume $\theta^{*}-$ Ind $X \leq n$ and suppose $x \in X$ and $\Psi=\left\{U_{\lambda}: \lambda \in \Lambda\right\}$ be an open cover of $X$

Since $X$ is $T_{1}$-space, then $\{x\}$ is closed set. By hypothesis there exists an open neighborhood $N$ of $\{x\}$ such that

$$
N \subset \bigcup_{\lambda \in \gamma} U_{\lambda}
$$

where $\gamma$ is a countably infinite subset of $\Lambda$ and $\theta^{*}-\operatorname{Ind} \partial(N) \leq n-1$. Using the induction hypothesis we obtain $\theta^{*}-\operatorname{ind} \partial(N) \leq n-1$. Therefore $\theta^{*}-$ Ind $X \leq n$.

Theorem 4.11 If $X$ is a compact space and $\theta^{*}-$ ind $X=0$, then $\theta^{*}-$ Ind $X=0$.

Proof Let $X$ be a compact space such that $\theta^{*}-$ ind $X=0$, let $Y$ be a closed set of $X$ and let $\Psi=\left\{U_{\lambda}: \lambda \in \Lambda\right\}$ be an open cover of $X$. Since $\theta^{*}-$ ind $X=0$, for each $x$ of $Y$ there exists an open- and - closed set $N_{x}$ such that

$$
x \in N_{x} \subset U_{\lambda \in \gamma} U_{\lambda}
$$

where $\gamma$ is countably infinite subset of $\Lambda$. Hence $\subset \cup_{x \in Y} N_{x} \subset \cup_{\lambda \in \gamma} U_{\lambda}$. Since $Y$ is closed in the compact space $X, Y$ is compact.

\section{References}

[1] D.S.Jankovisic, Ө-regular spaces, Internat.J.Math.Math.Sci.8(1985),no.3,615-619.

[2] M.M. Kovar, $\Theta$-regular spaces, Internat.J.Math.Math.Sci.17(1994),no.3,687-692.

[3] M.M. Kovar, -regular spaces, Internat.J.Math.Math.Sci.21(1998),no.1,199-200. CMP 1486 978.zbl 888.54027.

[4] B. Royy and M. N. Mukherjee" On a Type of Compactness Via Grills " MATEMATIQKI VESNIK 59 (2007), 113-120.

[5] B.Royand M. N. Mukherjee" A generalization of paracompactness in terms of grills" Math. Commun., Vol. 14(2009)

, No. 1, pp. 75-83.

[6] A.P. Pears,"On Dimension Theory of General Space", Cambridge University press(1975).

[7] J.R.Munkres,"Topology", Prentice Hall, upper saddle River 2000.

[8] S.Willard,"General Topology", AddisonWesty pub.Co., Inc. 1970. 
AL-Qadisiyah Journal of pure Science $\quad$ Vol.23 No.2 $\quad$ Year 2018

\section{بصض بديهيات الفصل الجديد مع تطبيقات في نظرية البعد \\ سمة كاظم جبر}

كلية الزراعة/قسم علوم التربة والموارد المائية

Semh.Alisawi@qu.ed.iq

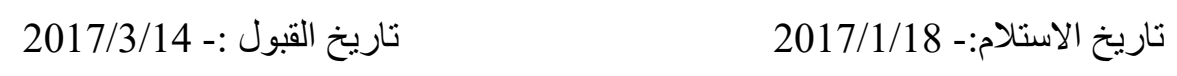

المستخلص

الهذف من هذا البحث هو تقديم ودراسة مفهومي الفضاء التبولوجي المنتظم نوع التطبيقات في نظرية البعد.

الكلمات المفتاحية. فضاء منتظم نوع- 